\title{
miRNA 与鼻咽癌发病机制
}

\author{
赵璐晴 ${ }^{(12)(3)}$, 陈雪 ${ }^{(1)(3)}$, 曹亚 ${ }^{(1)(2)}$ \\ (1) 教育部癌变与侵袭原理重点实验室, 长沙 410078; \\ (2) 卫生部癌变原理重点实验室, 长沙 410078; \\ (3) 中南大学湘雅医学院肿瘤研究所, 长沙 410078 \\ E-mail: xiaoqing1213@sina.com
}

2010-10-12 收稿, 2010-12-15 接受

国家重点基础研究发展计划(2011CB504300)和国家自然科学基金重点项目(30930101)资助

摘要 miRNA (microRNA) 是一族由 22 25 个核苷酸组成的非编码 RNA 小分子, 它通过与靶 mRNA 的 3'-非编码区以碱基互补配对的方式结合, 实现对靶基因转录后水平的降解或抑制. 最新研究表明, miRNA 与鼻咽癌中 EBV, LMP1, 信号转导通路, 肿瘤相关基因网络, 细胞有 丝分裂, 肿瘤血管生成和侵袭转移密切相关。因此, 探索 miRNA 与鼻咽癌发生发展之间的 关系, 有助于加深对其发病机制的认识, 也将为临床诊断和治疗提供新的启示与依据.

\section{关键词}

miRNA

鼻咽癌

EBV

LMP1

信号转导通路

有丝分裂

侵袭转移
miRNA (microRNA) 是近年来研究最热门的一种 生物小分子, 由 22 25 个核苷酸组成, 具有重要的生 物学功能. 随着研究工作的不断深人, 人们发现, 在 多种肿瘤组织中存在 miRNA 表达水平的异常, 这揭 示了肿瘤的发生发展与 miRNA 之间存在一定相关 性 ${ }^{[1]}$.

鼻咽癌是一种头颈部上皮来源的恶性肿瘤, 具 有高度的侵袭和转移特性, 好发于中国南部 ${ }^{[2]}$, 它的 发病与遗传易感性以及 EB 病毒 (Epstein-Barr virus, EBV) 感染有紧密联系 ${ }^{[3]}$. 最新研究 ${ }^{[4]}$ 显示, miRNA 与 鼻咽癌亦存在紧密的关联. 本文主要对 miRNA 与鼻 咽癌发病机制之间的关系予以评述.

\section{1 miRNA 的生成、作用机制及生物学功能}

\section{1 miRNA 的生成及作用机制}

miRNA 是一类长度为 $22 \sim 25$ 个核苷酸的内源性 非编码 RNA 小分子, 它的编码基因首先在 RNA 聚合 酶 II 作用下被转录为长度约几百个核苷酸的初级转 录物(pri-miRNAs), 然后在 RNA 聚合酶 III (Drosha 酶)
作用下被加工为具有茎环结构、长度为 60 70 个核苷 酸的 miRNAs 前体 (pre-miRNAs), 再由转运蛋白 (Exportin-5)识别并结合 pre-miRNAs 在 3'端的突出标 志, 依靠 Ran-GTP 酶将 pre-miRNAs 转运至细胞质; miRNAs 前体在 RNA 酶 III (Dicer 酶) 参与下被加工为 双链 miRNA, 在解旋酶作用下形成成熟的单链 miRNA; 单链 miRNA 进入核蛋白复合体, 参与形成 由 RNA 诱导的沉默复合体(miRNA-associated RNAinduced silencing complex, miRISC) ${ }^{[5 \sim 7]}$, 并通过此复 合体发挥生物学作用.

miRNA 主要通过与靶 mRNA 的 $3^{\prime}$ 端非编码区 (untranslated regions, UTRs)的碱基以完全或不完全 互补方式配对, 在转录后水平对靶 mRNA 进行降解 或抑制, miRNA 也参与基因转录后水平调控及多种 生物学功能发挥 ${ }^{[8,9]}$. 一般植物 miRNA 与靶基因完全 互补, 可使靶 mRNA 降解; 而动物 miRNA 与靶序列 的匹配程度较差, 不与靶基因完全互补, 只能抑制转 录后翻译 ${ }^{[10]}$. 许多 miRNA 与靶 mRNA 不完全互补, 因此转录后抑制是 miRNA 的主要作用模式 ${ }^{[11]}$. 一个 miRNA 可作用于多个靶 mRNA, 而一个靶 mRNA 可 
受多个 miRNA 调控, miRNA 与靶基因之间组成了复 杂的调控网络, 为基因表达水平的调控增加了一个 新的复杂的认识层面 ${ }^{[12]}$.

\section{2 miRNA 的生物学功能}

miRNA 的靶基因参与细胞生长、分化、增殖、 调亡、应激应答及干细胞分化潜能的维持等多种生物 学功能的发挥 ${ }^{[13]}$. miRNA 在肿瘤形成中也发挥重要 作用, miRNA 参与了肿瘤细胞的增殖、调亡、黏附、 血管生成等生物学过程的调控, 大约 50\%的 miRNA 位于肿瘤相关的染色体区域，在肿瘤发生的基因水 平调控方面发挥了广泛而重要的作用 ${ }^{[14]}$. 许多 miRNA 充当肿瘤抑制因子或致癌因子 ${ }^{[15,16]}$, 具有致 癌基因功能的 miRNA 通常被称为 OncomiR (致癌性 miRNA $)^{[17 \sim 19]}$, 它们包括 miR-155, miR-17-92 基因簇 (miR-17-5p, miR-17-3p, miR-18a, miR-19a, miR-20a, miR-19a- 1 和 miR-92-1) 和 miR-21 等; 而具有抑癌基 因功能的 miRNA 包括 miR-15a, miR-16-1, let-7 家族、 miR-143, miR-145 和 miR-34a 等 ${ }^{[20]}$. 最近, Douglas ${ }^{[21]}$ 将参与肿瘤转移的 miRNA 称为 metastamir (转移性 miRNA), 其中促进肿瘤转移的有 miR-10b, miR-21 等, 而抑制肿瘤转移的有 $\mathrm{miR}-141, \mathrm{miR}-200 \mathrm{a} / \mathrm{b} / \mathrm{c}$, miR-146 和 miR-335 等.

\section{2 miRNA 与鼻咽癌发病的分子机制}

\section{1 miRNA 与 $\mathrm{EBV}$}

$E B V$ 可编码多种 miRNA 基因 ${ }^{[22], ~}$ 它是第一个被 发现编码 miRNA 的病毒. 依据 miRBase ${ }^{[23]}$ 提供的数 据, 由 $E B V$ 编码的 miRNA 包括 ebv-miR- BHRF1-1 3 和 ebv-miR-BART1 22, 它们分别位于病毒基因组中 $B H R F 1$ 和 $B A R T$ 两个区域 ${ }^{[24]}$. 其中 $B H R F 1$ 为病毒复 制早期的基因, 在病毒裂解和复制水平升高时表达 增加, 与病毒裂解和复制的调控相关 ${ }^{[25]}$; 而 $B A R T^{[26]}$ 可编码病毒潜伏期的转录产物, 抑制病毒复制, 并使 复制保持在较低的水平.

$E B V$ 编码的 miRNA 参与调节病毒从裂解到潜伏 的转化, 也参与抵抗宿主对病毒的免疫反应 ${ }^{[27]}$. 这 些 miRNA 不仅作用于病毒本身的靶基因, 还作用于 宿主的靶基因 ${ }^{[28]}$. Xia 等人 ${ }^{[29]}$ 报道, miR-BHRF1-3 以 宿主的 CXCL11 为靶基因, CXCL11 的表达抑制将保 护被 $\mathrm{EBV}$ 感染的 $B$ 细胞免受细胞毒性 $T$ 细胞的破坏.
Choy 等人 ${ }^{[30]}$ 报道, miR-BART5 以宿主的 PUMA (p53upregulated modulator of apoptosis)为靶基因, PUMA 的下调将抑制被病毒感染的宿主细胞调亡.

Iizasa 等人 ${ }^{[31]}$ 报道, ebv-miR-BART6 的初级转录 产物 pri-miR-BART6 在被潜伏 EBV 感染的细胞中进 行编码, 成熟的 miR-BART6 在细胞多种潜伏阶段发 挥了重要作用. Iizasa 等人 ${ }^{[31]}$ 发现, miR-BART6-5p RNAs 能抑制 EBNA2 病毒癌基因的表达, 而这种癌 基因的表达在 I 型和 II 型潜伏状态(免疫低反应)向 III 型潜伏状态(免疫高反应)转化的过程中是不可或 缺的. Zta 和 Rta 病毒蛋白在 EBV 的裂解复制过程中 也发挥了重要作用 ${ }^{[32]}$. 这些结果揭示了 miR-BART6 在 EBV 的感染和潜伏中发挥了重要的调节作用.

上述研究表明, $E B V$ 编码的 miRNA 在鼻咽癌的 发生发展中发挥着重要作用, 它不仅作用于病毒本 身的靶基因, 有利于病毒的感染和潜伏; 也作用于宿 主的靶基因, 有利于病毒逃避宿主细胞的免疫反应 及抑制宿主细胞的调亡.

\section{2 miRNA 与 LMP1}

$E B V$ 编码的潜伏膜蛋白 1 (latent membrane protein 1, LMP1)是一种重要的病毒致瘤蛋白 ${ }^{[33]}$. LMP1 在 EBV 的 II 型和 III 型潜伏期产生，可调控 NF- $\mathrm{kB}$ 信 号转导通路及宿主细胞的生长和调亡. Natalie 等人 ${ }^{[34]}$ 发现, LMP1 可通过 NF- $\mathrm{kB}$ 途径激活 miR-146a 的启动 子, 诱导宿主细胞中 miR-146a 的表达; miR-146a 对 诱导或维持 $\mathrm{EBV}$ 的潜伏感染状态有重要作用, 可调 节 EBV 对宿主细胞的固有免疫反应 ${ }^{[35]}$. Graziana 等 人 $^{[36]}$ 也发现, LMP1 通过激活 NF- $\mathrm{KB}$ 途径来反式激活 miR-155 的转录, 上调 miR-155 的表达; miR-155 在淋 巴细胞的激活和 $\mathrm{EBV}$ 介导的 B 细胞转化中发挥关键 作用. PU. 1 是 miR-155 下游的靶基因, 在 miR-155 缺 陷的 B 细胞中高度表达, 可导致产生 $\mathrm{IgG} 1$ 的 B 细胞 数量减少, 提示 miR-155 在 B 细胞成熟过程中发挥了 关键作用 ${ }^{[36]}$.

Lo 等人 ${ }^{[37]}$ 报道, 有 3 种 $E B V$ 编码的 miRNA (miR-BART1-5p, miR-BART16 和 miR-BART17-5p) 可下调 LMP1 的表达. Zheng 等人 ${ }^{[38]}$ 发现, LMP1 引发 了 NF- $\mathrm{kB}, \mathrm{AP}-1$ 和 STAT 信号通路, 它通过上调两面 神激酶 3 (Janus kinase 3, JAK3)的表达和增加信号传 导及转录激活因子(signal transducers and activators of transcription, STAT)的磷酸化可激活 JAK/STAT 和 
PI-PLC-PKC 通路; 由 LMP1 诱发的连续级联的信号 转导瀑布的出现, 可产生一系列生物学效应, 最终导 致细胞周期的紊乱, 出现 $\mathrm{G} 1 / \mathrm{S}$ 期的加速及 $\mathrm{G} 2 / \mathrm{M}$ 期 的停滞; LMP1 还可诱导人端粒酶逆转录酶(human telomerase reverse transcriptase, hTERT)的表达, 促使 细胞永生化.

以上有关 miRNA 与 LMP1 的研究说明, $E B V$ 编码 的 miRNA 可调控 LMP1 的表达，同时 LMP1 可通过 信号转导通路激活 miRNA 的表达, 进而 miRNA 作用 于肿瘤相关的靶基因, 最终导致鼻咽癌的发生发展.

\section{3 miRNA 与鼻咽癌中信号转导通路及鼻咽癌 基因调控网络}

最近的研究显示, 多种不同 miRNA 可共同调节 同一条信号转导通路的相关靶基因表达 ${ }^{[39]}$. 因此, 对信号转导通路而非个别靶基因的研究, 将为研究 miRNA 的生物学功能提供新思路. Chen 等人 ${ }^{[40]}$ 发现, 在鼻咽癌的组织标本中 35 种 miRNA 的表达水平有 显著改变：显著上调的有 11 种，包括 miR-196b, miR138, miR-155, miR-142-3p 和 miR-18a 等; 显著下调 的有 24 种, 包括 miR-204, miR-449a, miR-34c-3p, miR-143 和 miR-145 等; 上调最明显的 4 种 miRNA 是 miR-142-3p, miR-196b, miR-138 和 miR-155; 下调 最明显的 4 种 miRNA 是 miR-204, miR-195, miR-187 和 miR-143. 表达下调的 miRNA 有 5 条重要的特异 性靶向通路: (1) G1/S 期转变的调节通路; (2) 血管内 皮生长因子(vascular endothelial growth factor, VEGF) 与血管生成蛋白- $1^{[41,42]}$ 之间对话的信号通路; (3) G 蛋 白(-)介导 MARK-ERK 信号通路的调节; (4) 转化生 成因子(transforming growth factor, TGF), WNT 信号 通路 ${ }^{[3]}$ 与细胞骨架的重构; (5) 膜依赖雌激素受体 1 (estrogen receptor 1, ESR1)与 G 蛋白相互作用的信号 通路. 其中 2 条最重要的靶向通路是 G1/S 期转变的 调节通路和 VEGF 与血管生成蛋白-1 之间对话的信 号通路. Chen 等人 ${ }^{[40]}$ 发现, 这 2 条通路中的 6 种靶基 因 (CCND2, CCND3, CDC25A, VEGFA, PLCG1 和 $A K T)$ 的表达水平在鼻咽癌组织中均上调, 即下调 miRNA 的靶基因在鼻咽癌中的表达上调. Chen 等 人 $^{[40]}$ 还发现, 下调 miRNA 的靶向信号通路参与了细 胞周期的调节、细胞生存与调亡的调控及细胞骨架的 重构. 上述研究表明, miRNA 的表达下调与相应靶基 因的表达上调之间反相关.
在鼻咽癌的发病机制中, miRNA 与原癌基因、抑 癌基因及相关靶基因共同组成了一个复杂的肿瘤形 成网络系统. Zhang 等人 ${ }^{[44]}$ 发现, miR-141 在鼻咽癌中 的表达上调, 且发挥原癌基因的作用; 通过敲除原癌 基因 $c-M Y C$ 或再表达抑癌基因 SPLUNCl, 可使 miR-141 的表达下调; miR-141 的表达下调可影响鼻 咽癌细胞的细胞周期, 细胞凋亡、生长、迁徙和转移. Zhang 等人 ${ }^{[44]}$ 通过苂光素酶报告基因及蛋白电泳发 现, BRD 3, UBAP1 及 PTEN 是 miR-141 的潜在作用靶 点; $\mathrm{BRD} 3$ 及 $\mathrm{UBAP}^{[45 \sim 47]}$ 参与鼻咽癌的发生发展, BRD3 也参与 $\mathrm{Rb} / \mathrm{E} 2 \mathrm{~F}$ 通路的调节; PTEN 在多种肿瘤 中充当重要的肿瘤抑制因子, 也参与 AKT 信号通路 的调节 ${ }^{[48]}$. 抑制 miR-141 的表达将影响 Rb/E2F, JNK2 及 $\mathrm{AKT}$ 通路中许多重要分子的功能 ${ }^{[44]}$. Zhang 等人 ${ }^{[4]}$ 认为, miR-141 和肿瘤相关基因 $c-M Y C, S P L U N C 1$, BRD3, UBAPI 及 PTEN 可能参与构建了一个“基因miRNA"网络系统, 进而导致鼻咽癌的发生发展.

上述研究表明, 在鼻咽癌的发生发展过程中, 一 系列遗传学及表观遗传学事件构成了复杂的网络系 统 ${ }^{[49 ~ 533}$. 原癌基因和抑癌基因均可调控 miRNA 的表 达，同时相应的 miRNA 表达可进一步调控肿瘤相关 靶基因的表达，因此 miRNA 与原癌基因、抑癌基因 和肿瘤相关靶基因共同构建了一个“基因-miRNA-基 因”结构的复杂网络调控系统, 从而促进了鼻咽癌的 发生发展过程.

\section{3 miRNA 在鼻咽癌发生发展中的生物学 功能}

\section{1 miRNA 与有丝分裂}

Plk1 是有丝分裂各阶段的一个关键调节因子 ${ }^{[54]}$, 在鼻咽癌的增殖和进展中也是一个重要的调节因子, Plk1 的表达异常与 miRNA 的表达下调相关 ${ }^{[55]}$. 作为 有丝分裂 $G 2 / M$ 期转化的关键调节因子, Plk1 在细胞 周期的调控中发挥重要作用 ${ }^{[56]}$. Wei 等人 ${ }^{[55]}$ 的研究发 现，在鼻咽癌中, miR-100 可调节 Plk1 的表达, siPlk1 (作用于 Plk1 的 siRNA, 此研究采用 miR-100)可改变 细胞结构并诱导异常纺锤体的形成, Plk1 的低表达可 促使癌细胞中多种碎裂的核碎片固缩 ${ }^{[57]}$ 及诱导异常 微管的形成. Wei 等人 ${ }^{[55]}$ 还发现, siPlk 能抑制 Plk1 的 mRNA 和蛋白质表达, siPlk1 与放疗结合可有效降低 C666-1 细胞的存活能力、增加磷酸化组蛋白 $\gamma \mathrm{H} 2 \mathrm{AX}$ 
的表达水平. siPlk1 也可通过诱导 G2/M 期的停滞, 增 强天冬氨酸特异性半胱氨酸蛋白酶 (cysteine-containing aspartate-specific protease, Caspase) 3/7 的活性 来诱发细胞调亡 ${ }^{[58]}$. 放疗可加速 G2/M 期停滞及细胞 调亡 ${ }^{[59]}$, 因此 siPlk1 ${ }^{[60]}$ 与放疗结合可抑制肿瘤生长.

在鼻咽癌中, miR-100 的表达下调可导致 Plk1 过 度表达, 从而导致鼻咽癌的发生发展. 因此, 以 Plk1 为靶点抑制 Plk1 的过度表达, 可促使有丝分裂崩解、 细胞周期停滞; 如果与放疗结合 ${ }^{[61,62]}$, 这种治疗策略 将非常有效.

\section{2 miRNA 与肿瘤的血管生成及侵袭转移}

血管生成广泛存在于生理学及病理学过程中, 它在机体生长、伤口愈合、炎症及肿瘤形成中发挥重 要作用 ${ }^{[63]}$. 许多小分子在血管生成过程中发挥正调 节作用, VEGF 是最重要的调控因子之一 ${ }^{[64]}$, 它的表 达受多种因素调控. Hua 等人 ${ }^{[65]}$ 发现, miR-16, miR20a, miR-20b, let-7b, miR-17-5p, miR-27a, miR-106a, miR-106b, miR-107, miR-193a, miR-210, miR-320, miR-361 和 miR-15b 等均可以 VEGF 为靶点, 调控 VEGF 的表达.

Olsson 等人 ${ }^{[66]}$ 发现, 在低氧刺激下, VEGF 与其 他血管生成因子的表达上调, 共同导致血管生成; 在 严格的时间、空间条件下, miRNA 和多种靶基因可共 同调节多种功能相关的基因及血管生成因子. Hua 等 人 ${ }^{[65]}$ 发现, miR-15b, miR-16, miR-20a 和 miR-20b 共 同作用于鼻咽癌细胞中的血管生成因子, 同时有 7 种 与血管生成相关的基因 $(V E G F, c-M E T, C O X 2, u P A R$, $P A I 1, M A P K 7$ 和 Ang) 在鼻咽癌细胞中的表达上调.

$\mathrm{Li}$ 等人 ${ }^{[67]}$ 发现, LMP1 作为一种促使鼻咽癌转移 的关键调节因子 ${ }^{[68]}$, 可诱导转录因子 Twist 的表达, 上调相应的 miR-10b 表达, 从而促使鼻咽癌转移; LMP1 也可通过激活多种信号转导通路, 来调节多种 转移相关基因(如 E-cadherin, MMPs, c-Met, VEGF, $E G F R, C O X-2)^{[69]}$ 的表达.

Sikumar 等人 ${ }^{[70]}$ 发现, 在鼻咽癌细胞中, miR-29c 的表达下调可导致下游多种靶基因的表达上调，而 这些靶基因编码的多种细胞外基质蛋白(如胶原蛋白 及层粘连蛋白 $\gamma 1$ )参与鼻咽癌细胞的侵袭和转移. 因 此, 可将鼻咽癌细胞中 miR-29c 的表达下调作为鼻咽 癌侵袭转移的特征. Xia 等人 ${ }^{[71]}$ 发现, miR-200a 作为 鼻咽癌发病机制中重要调节因子之一, 在鼻咽癌细 胞中的表达下调, 并介导下游功能性的靶基因 ZEB2
及 $C T N N B 1$ 表达下调, 从而不同程度地抑制鼻咽癌 细胞生长、迁徙和转移.

以上研究显示, 多种 miRNA及其靶基因与鼻咽癌 中的血管生成及侵袭转移密切相关 ${ }^{[72]}$, 这些 miRNA 与靶基因的共同作用将导致鼻咽癌细胞的侵袭和转移. 如何调节这些与转移相关 miRNA 的表达? 如何阻断 靶基因生物学功能的发挥? 这些都值得深人研究.

\section{4 与鼻咽癌相关的 miRNA 和未来研究展望}

综上所述, 表 1 中列举了已发现的各种 miRNA 与鼻咽癌之间的关系. 从表 1 可见, 近年来有关鼻咽 癌的发病机制与 miRNA 关系的研究获得了越来越多 的关注. 从 $E B V$ 编码的 miRNA 对宿主产生的生物学 效应, 到宿主细胞自身编码的 miRNA 在鼻咽癌发生 发展中发挥的生物学功能, 这些都引起了科学家们 的极大兴趣. 同时, 在对 miRNA 的研究中也出现了 许多值得深思的问题. 例如: (1) 在鼻咽癌的发病机 制中, 是否存在具有人核功能的 miRNA, 它能否发 挥转录因子的作用? (2) 在 miRNA 与靶基因作用的 邻近区域是否存在类似 Dead end 1 (Dnd1)的 RNA 结 合蛋白, 它能否间接抑制 miRNA 与靶点的相互作用? (3) 在鼻咽癌的诊断治疗中, 是否可在血清或体液中 检测到鼻咽癌高度特异性的 miRNA 表达, 它能否作 为一种生物标志物对鼻咽癌进行早期诊断? (4) 多种 miRNA 的表达是否可协同增加机体对放化疗的敏感 性? 这些都是今后需要重点研究的问题.

目前, 在有关鼻咽癌发病机制的研究中已发现 LMP1 与 miRNA 存在密切的联系, 因此今后可研究 LMP1 信号转导通路中 miRNA 与激酶、转录因子及 靶基因的相互作用等. LMP1 可通过调节信号转导通 路中 miRNA 的表达来实现对转移相关基因的调控, 因此对转移相关基因的特别关注将有助于深人了解 miRNA 在鼻咽癌侵袭转移中发挥的作用, 为减少鼻 咽癌的转移和复发提供新的靶点.

此外, Plk1 也是一个值得重点关注的靶分子. Plk1 在调节鼻咽癌细胞的有丝分裂及细胞周期中发 挥着非常关键的作用, 而且与放疗具有协同作用, 因 此深人研究 Plk1 相关的 miRNA 与放疗相结合的抑癌 机制, 将为鼻咽癌的治疗带来新的希望. 总之, 对 miRNA 与鼻咽癌发病机制之间关系的深人研究将为 我们认识鼻咽癌的发生发展提供更新的视角, 也将 为鼻咽癌的诊断和治疗带来新的思路与启示. 
表 1 与鼻咽癌相关的 miRNA

\begin{tabular}{|c|c|c|c|c|}
\hline miRNA & 靶点 & 生物学功能 & 在鼻咽癌中的表达情况 a) & 参考文献 \\
\hline ebv-miR-BART cluster 1 & LMP1 & 浸润、转移、永生化、凋亡、增殖 & $\uparrow$ & {$[37]$} \\
\hline ebv-miR-BART 6 & Dicer & EBV 的感染及潜伏状态 & $\uparrow$ & [31] \\
\hline ebv-miR-BART 5 & PUMA & 抑制细胞调亡 & $\uparrow$ & {$[30]$} \\
\hline ebv-miR-BHRF1-3 & CXCL11 & 免疫保护 & $\uparrow$ & [29] \\
\hline $\operatorname{miR}-141$ & BRD3, UBAP1, PTEN & 影响细胞周期、调亡、生长、迁徙、侵袭 & $\uparrow$ & {$[44]$} \\
\hline $\operatorname{miR}-29 c$ & 细胞外基质蛋白 & 侵袭、转移 & $\downarrow$ & {$[70]$} \\
\hline miR-200a & ZEB2, CTNNB1 & 细胞生长、迁徙、转移 & $\downarrow$ & [71] \\
\hline $\begin{array}{l}\operatorname{miR}-15 b, \text { miR-16, } \\
\text { miR-20a, miR-20b }\end{array}$ & VEGF & 影响血管生成 & $\downarrow$ & {$[65]$} \\
\hline $\begin{array}{l}\text { miR-204, miR-195, } \\
\text { miR-143, miR-187 }\end{array}$ & $\begin{array}{l}\text { CCND2, CCNE2, } \\
\text { CDC25A, VEGFA, } \\
\text { PLCG1, AKT }\end{array}$ & $\begin{array}{l}\text { 参与信号通路的调节及细胞周期、调亡、 } \\
\text { 细胞骨架的重构 }\end{array}$ & $\downarrow$ & {$[40]$} \\
\hline $\operatorname{miR}-100$ & Plk1 & 影响有丝分裂及细胞周期 & $\downarrow$ & [55] \\
\hline $\operatorname{miR}-155$ & PU.1 & $\begin{array}{l}\text { 促进细胞生长、分化、调亡, 参与淋巴 } \\
\text { 细胞的激活 }\end{array}$ & $\uparrow$ & {$[36]$} \\
\hline miR-10b & 未定 & 侵袭、转移 & $\uparrow$ & {$[67]$} \\
\hline
\end{tabular}

a) $\uparrow$, 高于正常鼻咽上皮组织中 miRNA 的水平; $\downarrow$, 低于正常鼻咽上皮组织中 miRNA 的水平

\section{参考文献}

1 George A C, Carlo M C. MicroRNA signatures in human cancers. Nat Rev Cancer, 2006, 6: 857-866

2 Chin D, Boyle G M, Porceddu S, et al. Head and neck cancer: Past, present and future. Expert Rev Anticancer Ther, 2006, 6: 1111-1118

3 Deyrup A T. Epstein-Barr virus-associated epithelial and mesenchymal neoplasms. Hum Pathol, 2008, 39: 473-483

4 Zhu J Y, Pfuhl T, Motsch N, et al. Identification of novel Epstein-Barr virus microRNA genes from nasopharyngeal carcinomas. J Virol, 2009, 83: 3333-3341

5 Georgia S. Emerging roles of microRNAs as molecular switches in the integrated circuit of the cancer cell. RNA, 2009, 15: 1443-1461

6 Filipowicz W, Bhattacharyya S N, Sonenberg N. Mechanisms of post-transcriptional regulation by microRNAs: Are the answers in sight? Nat Rev Genet, 2008, 9: 102-114

7 Israel A, Sharan R, Ruppin E, et al. Increased microRNA activity in human cancers. PLoS One, 2009, 4: e6045

8 Olson P, Lu J, Zhang H, et al. MicroRNA dynamics in the stages of tumorigenesis correlate with hallmark capabilities of cancer. Genes Dev, 2009, 23: 2152-2165

9 Zhang B, Pan X, Cobb G P, et al. MicroRNAs as oncogenes and tumor suppressors. Dev Biol, 2007, 302: 1-12

10 Wu M, Jolicoeur N, Li Z, et al. Genetic variations of microRNAs in human cancer and their effects on the expression of miRNAs. Carcinogenesis, 2008, 29: 1710-1716

11 Kumar M S, Lu J, Mercer K L, et al. Impaired microRNA processing enhances cellular transformation and tumorigenesis. Nature Genet, 2007, 39: 673-677

12 Liu C G, Calin G A, Volinia S, et al. MicroRNA expression profiling using microarrays. Nat Protoc, 2008, 3: 563-578

13 Hatfield S, Ruohola-Baker H. microRNA and stem cell function. Cell Tissue Res, 2008, 331: 57-66

14 Bushati N, Cohen S M. microRNA functions. Annu Rev Cell Dev Biol, 2007, 23: 175-205

15 Finoux A L, Chartrand P. Oncogenic and tumour suppressor microRNAs. Med Sci (Paris), 2008, 24: 1049-1054

16 Wang X, Tang S, Le S Y, et al. Aberrant expression of oncogenic and tumor-suppressive microRNAs in cervical cancer is required for cancer cell growth. PLoS One, 2008, 3: e2557

17 Aurora E K, Frank J S. Oncomirs-microRNAs with a role in cancer. Nat Rev Cancer, 2006, 6: 259-269

18 Rai D, Karanti S, Jung I, et al. Coordinated expression of microRNA-155 and predicted target genes in diffuse large B-cell lymphoma. Cancer Genet Cytogenet, 2008, 181: 8-15

19 Takakura S, Mitsutake N, Nakashima M, et al. Oncogenic role of miR-17-92 cluster in anaplastic thyroid cancer cells. Cancer Sci, 2008, 99: 1147-1154

20 Welch C, Chen Y, Stallings R L. MicroRNA-34a functions as a potential tumor suppressor by inducing apoptosis in neuroblastoma cells. Oncogene, 2007, 26: 5017-5022

21 Douglas R H. Metastamir: The field of metastasis-regulatory microRNA is spreading. Cancer Res, 2009, 69: 7495-7498 
22 Cosmopoulos K, Pegtel M, Hawkins J, et al. Comprehensive profiling of Epstein-Barr virus microRNAs in nasopharyngeal carcinoma. J Virol, 2009, 83: 2357-2367

23 Griffiths-Jones S, Saini H K, van Dongen S, et al. miRBase: Tools for microRNA genomics. Nucleic Acids Res, 2008, 36: D154-D158

24 Gourzones C, Gelin A, Bombik I, et al. Extra-cellular release and blood diffusion of BART viral micro-RNAs produced by EBV-infected nasopharyngeal carcinoma cells. Virol J, 2010, 7: 271

25 Jing Y Z, Wang Y, Jia Y P, et al. Polymorphisms of Epstein-Barr virus BHRF1 gene, a homologue of bcl-2. Chin J Cancer, 2010, 29: $1000-1005$

26 Edwards R H, Marquitz A R, Raab-Traub N. Epstein-Barr virus BART microRNAs are produced from a large intron prior to splicing. J Virol, 2008, 82: 9094-9106

27 Ghosh Z, Mallick B, Chakrabarti J. Cellular versus viral microRNAs in host-virus interaction. Nucleic Acids Res, 2009, 37: 1035-1048

28 Cullen B R. Viral and cellular messenger RNA targets of viral microRNAs. Nature, 2009, 457: 421-425

29 Xia T, O'Hara A, Araujo I, et al. EBV microRNAs in primary lymphomas and targeting of CXCL-11 by ebv-mir-BHRF1-3. Cancer Res, 2008, 68: 1436-1442

30 Choy E Y, Siu K L, Kok K H, et al. An Epstein-Barr virus-encoded microRNA targets PUMA to promote host cell survival. J Exp Med, 2008, 205: 2551-2560

31 Iizasa H, Wulff B E, Alla N R, et al. Editing of Epstein-Barr virus-encoded BART6 microRNAs controls their dicer targeting and consequently affects viral latency. J Biol Chem, 2010, 285: 33358-33370

32 Chang L K, Chuang J Y, Nakao M, et al. MCAF1 and synergistic activation of the transcription of Epstein-Barr virus lytic genes by Rta and Zta. Nucleic Acids Res, 2010, 38: 4687-4700

33 Hariwiyanto B, Sastrowiyoto S, Mubarika S, et al. LMP1 and LMP2 may be prognostic factors for outcome of therapy in nasopharyngeal cancers in Indonesia. Asian Pac J Cancer Prev, 2010, 11: 763-766

34 Natalie M, Thorsten P, Jan M, et al. Epstein-Barr virus-encoded latent membrane protein 1 (LMP1) induces the expression of the cellular microRNA miR-146a. RNA Biol, 2007, 4: 131-137

35 Middeldorp J M, Pegtel D M. Multiple roles of LMP1 in Epstein-Barr virus induced immune escape. Semin Cancer Biol, 2008, 18: 388-396

36 Graziana G, Annalisa R, Daniela R, et al. Epstein-Barr virus latent membrane protein 1 trans-activates miR-155 transcription through the NF-kB pathway. Nucleic Acids Res, 2008, 36: 6608-6619

37 Lo A K, To K F, Lo K W, et al. Modulation of LMP1 protein expression by EBV-encoded microRNAs. Proc Natl Acad Sci USA, 2007, 104: $16164-16169$

38 Zheng H, Li L L, Hu D S, et al. Role of Epstein-Barr virus encoded latent membrane protein 1 in the carcinogenesis of nasopharyngeal carcinoma. Cell Mol Immunol, 2007, 4: 185-196

39 Liu Q, Fu H, Sun F, et al. miR-16 family induces cell cycle arrest by regulating multiple cell cycle genes. Nucleic Acids Res, 2008, 36 : 5391-5404

40 Chen H C, Chen G H, Chen Y H, et al. MicroRNA deregulation and pathway alterations in nasopharyngeal carcinoma. Br J Cancer, 2009, 100: $1002-1011$

41 Li Y H, Hu C F, Shao Q, et al. Elevated expressions of survivin and VEGF protein are strong independent predictors of survival in advanced nasopharyngeal carcinoma. J Transl Med, 2008, 6: 1

42 Pan J, Kong L, Lin S, et al. The clinical significance of coexpression of cyclooxygenases-2, vascular endothelial growth factors, and epidermal growth factor receptor in nasopharyngeal carcinoma. Laryngoscope, 2008, 118: 1970-1975

43 Zeng Z Y, Zhou Y H, Zhang W L, et al. Gene expression profiling of nasopharyngeal carcinoma reveals the abnormally regulated Wnt signaling pathway. Hum Pathol, 2007, 38: 120-133

44 Zhang L M, Deng T, Li X Y, et al. MicroRNA-141 is involved in a nasopharyngeal carcinoma-related genes network. Carcinogenesis, 2010, 31: 559-566

45 Zhou H D, Li X L, Li G Y, et al. Effect of SPLUNC1 protein on the Pseudomonas aeruginosa and Epstein-Barr virus. Mol Cell Biochem, 2008, 309: 191-197

46 Nakada C, Matsuura K, Tsukamoto Y, et al. Genome-wide microRNA expression profiling in renal cell carcinoma: Significant down-regulation of miR-141 and miR-200c. J Pathol, 2008, 216: 418-427

47 Xiao B, Fan S, Zeng Z, et al. Purification of novel UBAP1 protein and its decreased expression on nasopharyngeal carcinoma tissue microarray. Protein Expr Purif, 2006, 47: 60-67

48 Chalhoub N, Baker S J. PTEN and the PI3-kinase pathway in cancer. Annu Rev Pathol, 2009, 4: 127-150

49 Shen G P, Pan Q H, Hong M H, et al. Human genetic variants of homologous recombination repair genes first found to be associated with Epstein-Barr virus antibody titers in healthy cantonese. Int J Cancer, 2010, doi: 10.1002/ijc.25759 
50 Ran Y, Wu S, You Y. Demethylation of E-cadherin gene in nasopharyngeal carcinoma could serve as a potential therapeutic strategy. J Biochem, 2010, doi: 10.1093/jb/mvq128

51 Guo Y, Chen J X, Yang S, et al. Selection of reliable reference genes for gene expression study in nasopharyngeal carcinoma. Acta Pharmacol Sin, 2010, 31: 1487-1494

$52 \mathrm{Xu} \mathrm{Y} \mathrm{F,} \mathrm{Liu} \mathrm{W} \mathrm{L,} \mathrm{Dong} \mathrm{J} \mathrm{Q,} \mathrm{et} \mathrm{al.} \mathrm{Sequencing} \mathrm{of} \mathrm{DC-SIGN} \mathrm{promoter} \mathrm{indicates} \mathrm{an} \mathrm{association} \mathrm{between} \mathrm{promoter} \mathrm{variation} \mathrm{and} \mathrm{risk} \mathrm{of} \mathrm{na-}$ sopharyngeal carcinoma in Cantonese. BMC Med Genet, 2010, doi: 10.1186/1471-2350-11-161

53 Wang S, Xiao X, Zhou X, et al. TFPI-2 is a putative tumor suppressor gene frequently inactivated by promoter hypermethylation in nasopharyngeal carcinoma. BMC Cancer, 2010, doi: 10.1186/1471-2407-10-617

54 Strebhardt K, Ullrich A. Targeting Polo-like kinase 1 for cancer therapy. Nat Rev Cancer, 2006, 6: 321-330

55 Wei S, Nehad M A, Carlo B, et al. Significance of Plk1 regulation by miR-100 in human nasopharyngeal cancer. Int J Cancer, 2010, 126: 2036-2048

56 Liu X, Lei M, Erikson R L. Normal cells, but not cancer cells, survive severe Plk1 depletion. Mol Cell Biol, 2006, 26: 2093-2108

57 Jang Y J, Ji J H, Choi Y C, et al. Regulation of Polo-like kinase 1 by DNA damage in mitosis: Inhibition of mitotic PLK-1 by protein phosphatase 2A. J Biol Chem, 2007, 282: 2473-2482

58 Sun F, Mikuni S, Kinjo M. Monitoring the caspase cascade in single apoptotic cells using a three-color fluorescent protein substrate. Biochem Biophys Res Commun, 2010, doi: 10.1016/j.bbrc.2010.12.047

59 Syljuasen R G, Jensen S, Bartek J, et al. Adaptation to the ionizing radiation-induced G2 checkpoint occurs in human cells and depends on checkpoint kinase 1 and Polo-like kinase 1 kinases. Cancer Res, 2006, 66: 10253-10257

60 Spankuch B, Kurunci-Csacsko E, Kaufmann M, et al. Rational combinations of siRNAs targeting Plk1 with breast cancer drugs. Oncogene, 2007, 26: 5793-5807

61 Su S F, Han F, Zhao C, et al. Long-term outcomes of early-stage nasopharyngeal carcinoma patients treated with intensity-modulated radiotherapy alone. Int J Radiat Oncol Biol Phys, 2010, doi: 10.1016/j.ijrobp.2010.09.011

62 Zhao L, Wan Q, Zhou Y, et al. The role of replanning in fractionated intensity modulated radiotherapy for nasopharyngeal carcinoma. Radiother Oncol, 2010, doi: 10.1016/j.radonc.2010.10.009

63 Shai E, Varon D. Development, cell differentiation, angiogenesis-Microparticles and their roles in angiogenesis. Arterioscler Thromb Vasc Biol, 2011, 31: 10-14

64 Lv X, Xiang Y Q, Cao S M, et al. Prospective validation of the prognostic value of elevated serum vascular endothelial growth factor in patients with nasopharyngeal carcinoma: More distant metastases and shorter overall survival after treatment. Head Neck, 2010, doi: 10.1002/hed.21541

65 Hua Z, Lv Q, Ye W B, et al. MiRNA-directed regulation of VEGF and other angiogenic factors under hypoxia. PLoS One, 2006, 1: e116

66 Olsson A K, Dimberg A, Kreuger J, et al. VEGF receptor signalling-in control of vascular function. Nat Rev Mol Cell Biol, 2006, 7: 359-371

67 Li G, Wu Z R, Peng Y, et al. MicroRNA-10b induced by Epstein-Barr virus-encoded latent membrane protein-1 promotes the metastasis of human nasopharyngeal carcinoma cells. Cancer Lett, 2010, 299: 29-36

68 Chew M M, Gan S Y, Khoo A S, et al. Interleukins, laminin and Epstein-Barr virus latent membrane protein 1 (EBV LMP1) promote metastatic phenotype in nasopharyngeal carcinoma. BMC Cancer, 2010, 10: 574

69 Kim T J, Lee Y S, Kang J H, et al. Prognostic significance of expression of vegf and cox-2 in nasopharyngeal carcinoma and its association with expression of C-erbB2 and EGFR. J Surg Oncol, 2010, doi: 10.1002/jso.21767

70 Sikumar S, Johan A B, Chen I H, et al. MicroRNA 29c is down-regulated in nasopharyngeal carcinomas, up-regulating mRNAs encoding extracelluar matrix proteins. Proc Natl Acad Sci USA, 2008, 105: 5874-5878

71 Xia H, Ng S S, Jiang S, et al. MiR-200a-mediated downregulation of ZEB2 and CTNNB1 differentially inhibits nasopharyngeal carcinoma cell growth, migration and invasion. Biochem Biophys Res Commun, 2010, 391: 535-541

72 Chen L C, Chen C C, Liang Y, et al. A novel role for TNFAIP2: Its correlation with invasion and metastasis in nasopharyngeal carcinoma. Mod Pathol, 2010, doi: 10.1038/modpathol.2010.193 